\title{
Helical Structure of Oligo- and Poly(m-substituted phenyl isocyanate)s Bearing an Optically Active End-Group
}

\author{
Katsuhiro MaEDa and Yoshio Окамото ${ }^{\dagger}$ \\ Department of Applied Chemistry, \\ Graduate School of Engineering, Nagoya University, \\ Furo-cho, Chikusa-ku, Nagoya 464-01, Japan
}

(Received May 19, 1997)

\begin{abstract}
Oligomerization of phenyl isocyanate derivatives, such as $m$-methoxyphenyl isocyanate ( $m$ MeOPI), 3,5 -dimethylphenyl isocyanate $(3,5 \mathrm{MePI})$, and $m$-chlorophenyl isocyanate $(m \mathrm{CIPI})$, bearing a substituent at meta position was carried out by using the lithium amide (Li- $(-)-\mathrm{MMP})$ of $(S)-(-)$-2-(methoxymethyl)pyrrolidine as an initiator to obtain the oligomers containing an optically active group at the initial chain end ( $\alpha$-end). When the reaction was terminated with $\mathrm{HCl}$-methanol, the oligomers of various degree of polymerization (DP) were isolated for $m \mathrm{MeOPI}$ and $3,5 \mathrm{MePI}$, but were not for $m$ CIPI. However, oligo $(m$ ClPI $)$ s were obtained when the reaction was terminated with acetic anhydride to introduce an acetyl group at the $\omega$-end. The obtained oligomers were separated in terms of DP using supercritical fluid chromatography (SFC) and the specific rotation of each oligomer was estimated. The highest specific rotation $[\alpha]_{365}^{25}$ was observed at DP $=13-14$ for oligo $(m \mathrm{MeOPI}), \mathrm{DP}=15$ for oligo(3,5MePI), and $\mathrm{DP}=10$ for oligo( $m \mathrm{ClPI})$. These results suggest that the one-handed helical structure can persist to a longer distance as the electron-donating power of the meta substituents increases.

KEY WORDS Oligomer / Aryl Isocyanate / Optically Active Polymer / Helix / Anionic Polymerization Supercritical Fluid Chromatography /
\end{abstract}

Poly(alkyl isocyanate)s are known to have a dynamic helical structure in solution due to the partial double bond character of a main chain and the steric repulsion between main chain carbonyl groups and side chains. ${ }^{1-3}$ Since helix reversals of the polymer chain occur extremely fast, the asymmetric synthesis of an optically active polyisocyanate possessing a rigid completely singlehanded helical structure has not yet been achieved. However, the introduction of a small amount of a chiral component into a polymer chain can induce a prevailing helical structure for poly(alkyl isocyanate)s because of the long persistence length of the helices. Such examples can be seen for the copolymers of an achiral isocyanate with a small amount of an optically active isocyanate, ${ }^{4-6}$ and for the polymers obtained with an optically active anionic initiator. ${ }^{7}$ Poly(alkyl isocyanate)s are also known to exist in a predominantly one-handed helical conformation in optically active solvents. ${ }^{8,9}$

On the other hand, poly(aryl isocyanate)s had been considered to have a random coil conformation due to the lack of stiffness in the main chain compared with poly(alkyl isocyanate)s. However, we recently found that achiral aryl isocyanates also can afford optically active polymers in the polymerization with chiral anionic initiators. ${ }^{10}$ This indicates that poly(aryl isocyanate)s as well as poly(alkyl isocyanate)s are able to have a helical structure in solution. We also reported that the helix reversal of poly( $m$-methylphenyl isocyanate) (poly$(m \mathrm{MePI})$ ) with a chiral group at the $\alpha$-end begins to occur at $\mathrm{DP}=13$ through the chiroptical study on the oligomers. ${ }^{11}$ However, much less study on the helical structure of poly(aryl isocyanate)s ${ }^{11,12}$ have been done in comparison with poly(alkyl isocyanate)s. ${ }^{13-16}$ In the present work, we synthesized the oligomers of meta substituted phenyl isocyanates (Scheme 1) possessing

† To whom all correspondence should be addressed. an optically active $(S)$-2-(methoxymethyl)pyrrolidinyl group at the $\alpha$-end. The obtained oligomer mixture was fractionated in terms of DP and the specific rotation of each oligomer was estimated in order to investigate the substituent effect on the helical structure of poly(aryl isocyanate)s.

\section{EXPERIMENTAL}

Materials

(S)-(-)-2-(Methoxymethyl)pyrrolidine ((-)-MMP) (Aldrich) $\left([\alpha]_{365}^{25}-9.9^{\circ},[\alpha]_{\mathrm{D}}^{25}+2.3^{\circ}(c 2.6\right.$, benzene $\left.)\right)$ was dried over $\mathrm{CaH}_{2}$ and then distilled under reduced pressure. $m$-Methoxyphenyl isocyanate (Aldrich), 3,5dimethylphenyl isocyanate (Aldrich), and $m$-chlorophenyl isocyanate (Aldrich) were also dried over $\mathrm{CaH}_{2}$ and distilled under reduced pressure just before use. Tetrahydrofuran (THF) (Wako) was purified by distillation from $\mathrm{Na}$ onto $\mathrm{CaH}_{2}$ followed by distillation onto $\mathrm{LiAlH}_{4}$ under high vacuum and then distilled again under high vacuum just before use. Acetic anhydride (Kishida) was purified by distillation. $t$-Butyllithium (Aldrich) was used as purchased and stored in an glass ampule under nitrogen atmosphere.

\section{Preparation of Initiator Solution}

Lithium amide of (-)-MMP (Li-(-)-MMP) was prepared by adding an equimolar amount of $t$-butyllithium in pentane to a solution of (-)-MMP in THF at ambient temperature just before use.

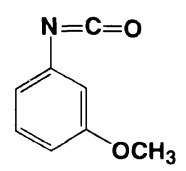<smiles>Cc1cc(C)cc(N=C=O)c1</smiles><smiles>O=C=Nc1cccc(Cl)c1</smiles>

Scheme 1. 


\section{Oligomerization Procedure}

Oligomerization was carried out in a glass ampule under a dry nitrogen atmosphere, where THF and the monomer were placed and then cooled to $-98^{\circ} \mathrm{C}$. The oligomerization was initiated by adding the $\mathrm{Li}-(-)$-MMP solution to the monomer solution with a syringe. After $4 \mathrm{~h}$, the reaction was terminated by adding ten fold excess $\mathrm{HCl}$ in methanol or acetic anhydride $\left(\mathrm{Ac}_{2} \mathrm{O}\right)$ in THF to the initiator. In the case of the termination with $\mathrm{Ac}_{2} \mathrm{O}$, the termination reaction was carried out under dry nitrogen atmosphere and the reaction mixture was kept for $1 \mathrm{~h}$ at $-78^{\circ} \mathrm{C}$ and then for $16 \mathrm{~h}$ at room temperature after adding $\mathrm{Ac}_{2} \mathrm{O}$ solution. The mixture was poured into a large amount of methanol and the polymer precipitated was separated by centrifugation. The oligomers collected as the methanol-soluble part was concentrated by evaporation under reduced pressure, dissolved in chloroform, and washed with water. The chloroform layer was dried over anhydrous magnesium sulfate and then evaporated under reduced pressure to recover the oligomers. The oligomers terminated with $\mathrm{Ac}_{2} \mathrm{O}$ were dissolved in dimethylsulfoxide (DMSO) and stirred for $12 \mathrm{~h}$ at room temperature in order to remove the oligomers that had not been end-capped with an acetyl group. It has been confirmed that in DMSO the end-uncapped polymer easily depolymerized by accompanying with the formation of cyclic trimer within a few hours. The DMSO solution was poured into a large amount of hexane-ethanol $(3: 1)$ in order to remove the depolymerization products. The linear oligomers precipitated were collected by centrifugation and dried in vacuo.

\section{Fractionation of Oligomers}

The oligomers were fractionated in terms of DP by supercritical fluid chromatography (SFC). SFC was performed on a JASCO 880-PU chromatograph equipped with a JASCO 875-UV UV detector on a silica gel column $(25 \times 1.0$ (i.d.) $\mathrm{cm})$ using $\mathrm{CO}_{2}$-ethanol containing $0.1 \%$ acetic acid as the mobile phase. Back-pressure was kept at $205 \mathrm{kgfcm}^{-2}$ by a JASCO $880-81$ backpressure regulator. Column temperature was maintained at $40^{\circ} \mathrm{C}$ with a JASCO $888-\mathrm{CO}$ column oven. The composition of the mobile phase was controlled as follows; $\mathrm{CO}_{2} / \mathrm{EtOH}=92 / 8(0 \rightarrow 10 \mathrm{~min}), 92 / 8 \rightarrow 68 / 32(10 \rightarrow 75 \mathrm{~min})$. The structure and DP of the oligomers were determined by ${ }^{1} \mathrm{H}$ NMR and FAB mass analyses.

\section{Measurement \\ ${ }^{1} \mathrm{H}$ NMR spectra were measured at $60^{\circ} \mathrm{C}$ in $\mathrm{CDCl}_{3}$ using a Varian VXR-500 $(500 \mathrm{MHz})$ or Gemini-2000 $(400 \mathrm{MHz})$ spectrometer with tetramethylsilane as an internal standard. FAB mass spectra were taken using $m$-nitrobenzyl alcohol $(m$-NBA) as a matrix with a JEOL JMS-AX505HA spectrometer. Optical Rotation was measured on a JASCO DIP-181 polarimeter. The molecular weight of the polymer was determined by GPC measurement on a Shodex System-21 GPC system equipped with an RI detector using THF as an eluent. Two GPC columns Shodex KF-803 and Shodex KF 806L were connected in series. Molecular weight calibration curves were obtained using seven polystyrene standards. The GPC measurement of the oligomers was performed}

Table I. Oligomerization of meta substituted phenyl isocyanate derivatives with $\mathrm{Li}-(-)$-MMP in THF at $-98^{\circ} \mathrm{C}^{\mathrm{a}}$

\begin{tabular}{cccccc}
\hline & & \multicolumn{4}{c}{ MeOH insoluble part } \\
\cline { 3 - 6 } & & Yield $(\%)^{\mathrm{b}}$ & {$[\alpha]_{365}^{25}{ }^{\mathrm{c}}$} & $M_{n}\left(\times 10^{3}\right)^{\mathrm{d}}$ & $M_{w} / M_{n}{ }^{\mathrm{d}}$ \\
\hline 1 & $m$ MeOPI & 32 & $+1670^{\circ} \mathrm{e}$ & 2.9 & 1.4 \\
2 & $3,5 \mathrm{MePI}$ & 5 & n.d. $^{\mathrm{f}}$ & n.d. & n.d. \\
3 & $m$ ClPI & 0 & - & - & -
\end{tabular}

${ }^{\text {a }}$ Monomer $0.5 \mathrm{~g}$, THF $5 \mathrm{ml},[\mathrm{M}] /[\mathrm{I}]=10$, time $4 \mathrm{~h}$, terminated with HCl-methanol. ${ }^{\mathrm{b}}$ Based on monomer. ${ }^{\mathrm{c}}$ In THF. ${ }^{\mathrm{d}}$ Determined by GPC using polystyrene standard in THF. ${ }^{\mathrm{e}}[\alpha]_{\mathrm{D}}^{25}+373^{\circ} .{ }^{\mathrm{f}}$ Insoluble in THF and $\mathrm{CHCl}_{3}$

on a JASCO 880-PU equipped with a JASCO 875-UV UV detector and a Shodex OR-1 polarimeter detector using $\mathrm{CHCl}_{3}$ as an eluent. Two JASCO Finepak GEL S101C columns connected in series were used.

\section{RESULTS AND DISCUSSION}

The oligomerization reaction initiated with $\mathrm{Li}-(-)-$ MMP followed by termination with $\mathrm{HCl}$-methanol afforded the polymers as a methanol-insoluble fraction for $m \mathrm{MeOPI}$ and 3,5MePI bearing electron-donating substituent(s), but did not for $m$ ClPI bearing an electron-withdrawing substituent as shown in Table I. Figure 1 depicts the GPC curves of the methanol-soluble part (oligomers) of $m$-substituted phenyl isocyanates (Scheme 2). Oligomers of various DP were observed for $m \mathrm{MeOPI}$ and 3,5MePI, while only oligomers up to the 3-mer were observed for $m$ ClPI. As for $m \mathrm{ClPI}$, the methanol-soluble part consisted mainly of the cyclic trimer, which indicates that linear oligomers may be unstable and depolymerized accompanying with the formation of the cyclic trimer during the oligomerization reaction or in the work-up process.

Figure 2 shows the SFC chromatograms of the methanol-soluble oligomers of $m \mathrm{MeOPI}$ and 3,5MePI performed on a silica gel column. In the both separations, the elution time of the oligomers except for the 1 -mer increased with an increase in DP. In addition, only both 1-mers showed negative optical rotation, whereas the other higher oligomers showed positive optical rotation. This may be ascribed to the fact that the 1-mer is impossible to have a helical conformation which can contribute the optical activity. The content of the 3mers was much higher than that of the other oligomers. This is probably because there exists a high energy barrier in the propagation step from a 3-mer anion to a 4-mer anion or the oligomers of low DPs more readily depolymerize to the 3-mer accompanying with the formation of cyclic trimers than those of high DPs. Figure 3 demonstrates ${ }^{1} \mathrm{H} \quad \mathrm{NMR}$ spectra of the 6-mer of $m \mathrm{MeOPI}$ and the 12-mer of 3,5MePI isolated. The spectra are reasonably assigned to each oligomer. The oligomers were stable enough to be isolated by SFC.

The specific rotation of the isolated oligomers are plotted against DP (Figure 4). The specific rotation of the higher oligomers ( $D P \geq 5$ ) were estimated from the peak area ratio of the GPC chromatograms monitored with polarimeter and UV detectors as exemplified in 


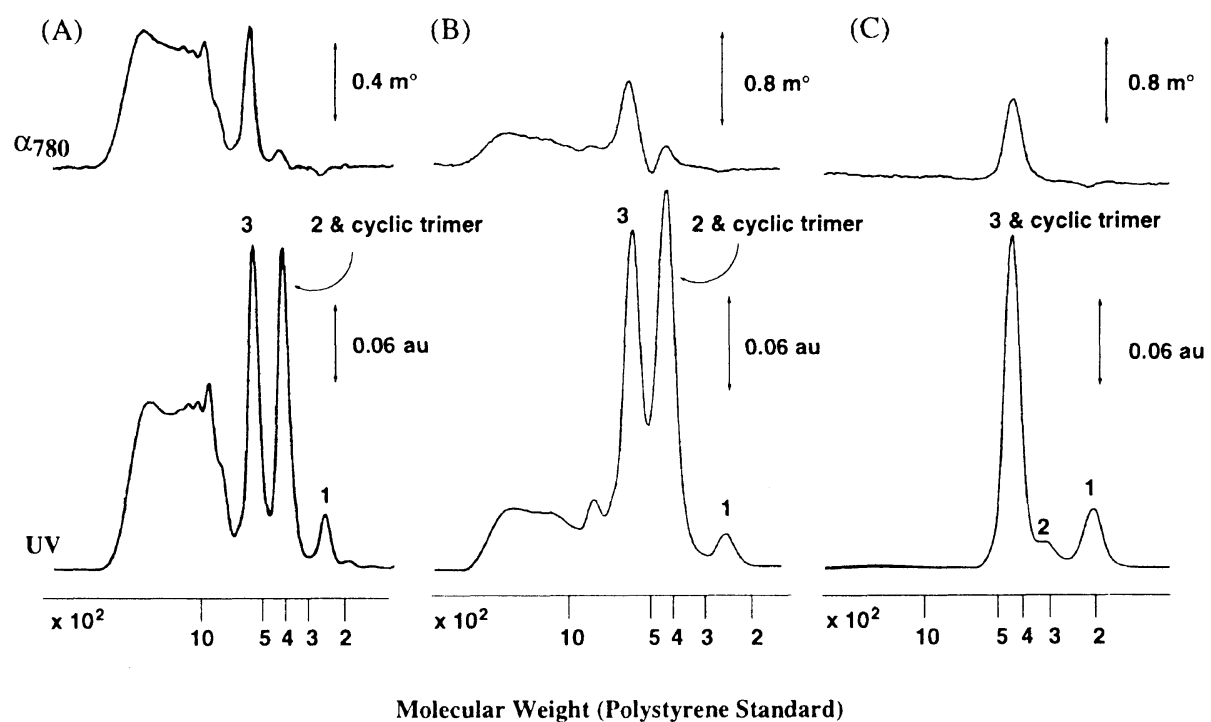

Figure 1. GPC curves of oligo $(m \mathrm{MeOPI})(\mathrm{A})$, oligo(3,5MePI) (B), and oligo $(m \mathrm{ClPI})(\mathrm{C})$ measured with $\mathrm{UV}(254 \mathrm{~nm})($ bottom $)$ and polarimeter $(780 \mathrm{~nm})$ (top) detectors.

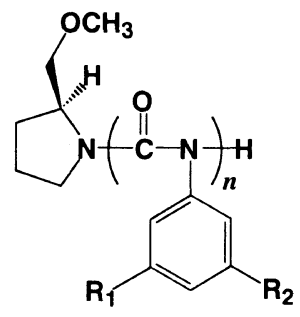

Oligo $(m \mathrm{MeOPI}): \mathbf{R}_{1}=\mathrm{OMe}, \mathbf{R}_{2}=\mathbf{H}$

Oligo(3,5MePI) $: \mathbf{R}_{1}=\mathrm{Me}, \mathbf{R}_{2}=\mathrm{Me}$

$\operatorname{Oligo(mCIPI)} \quad: \mathbf{R}_{1}=\mathrm{Cl}, \mathbf{R}_{2}=\mathbf{H}$<smiles>O=c1n(-c2cccc(Cl)c2)c(=O)n(-c2cccc(Cl)c2)c(=O)n1-c1cccc(Cl)c1</smiles>

Cyclic Trimer of $m$ CIPI

Scheme 2.
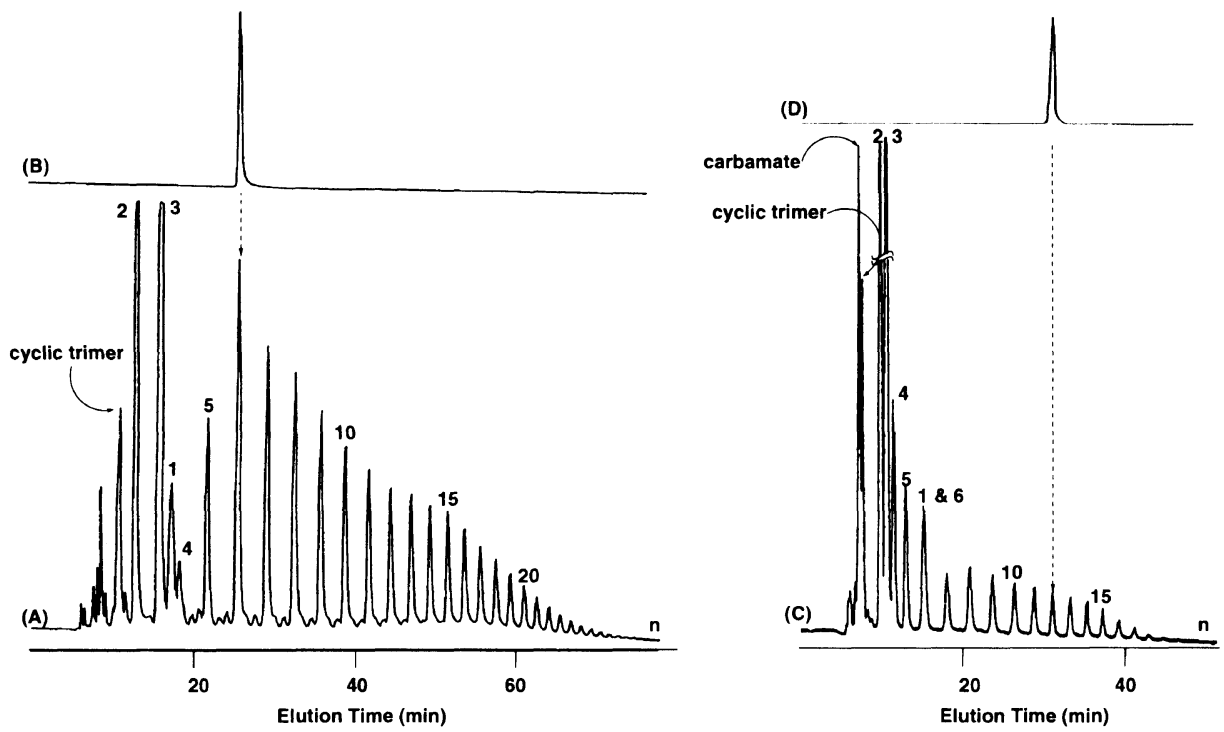

Figure 2. SFC curves of oligo $(m \mathrm{MeOPI})(\mathrm{A})$, 6-mer isolated from (A) (B), oligo(3,5MePI) (C), and 12-mer isolated from (C) (D) measured with UV $(254 \mathrm{~nm})$ detector.

Figure 5. The specific rotation of the oligomers of $m \mathrm{MeOPI}$ and 3,5MePI increased with an increase in DP in the range of $\mathrm{DP} \leq 13$ and $\mathrm{DP} \leq 15$, respectively. As described in the previous report, ${ }^{11}$ this is probably because in these DP ranges the oligomers have no helix reversal point and the helical structure becomes stiffer as the DP increases because the vicinity of polymer chain ends should have a higher mobility than the interior of a polymer chain and this effect of the chain ends should be reduced as the DP increases. The gradual decrease of 


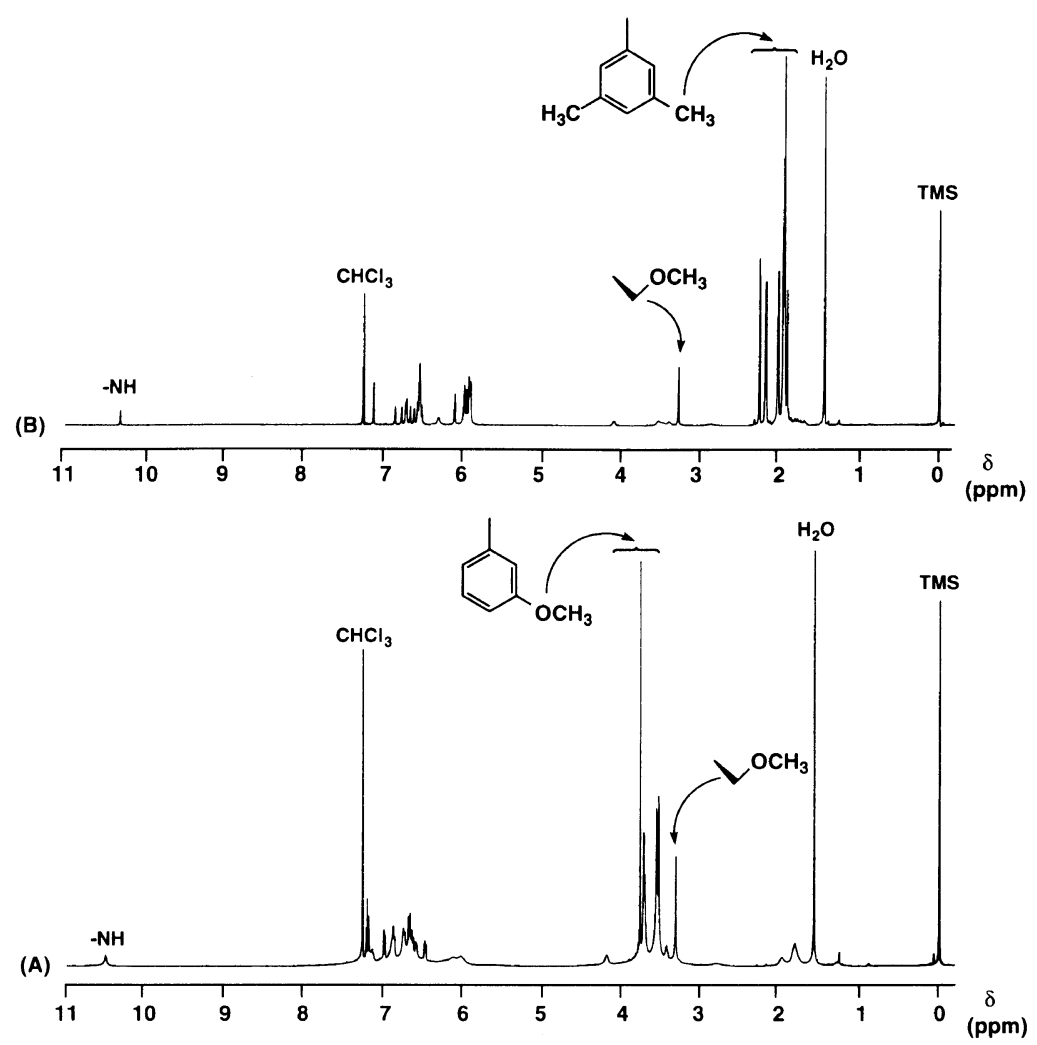

Figure 3. ${ }^{1} \mathrm{H}$ NMR spectra of 6-mer of $m \mathrm{MeOPI}(\mathrm{A})$ and 12 -mer of $3,5 \mathrm{MePI}(\mathrm{B})$ in $\mathrm{CDCl}_{3}$ at $60^{\circ} \mathrm{C}(500 \mathrm{MHz})$.

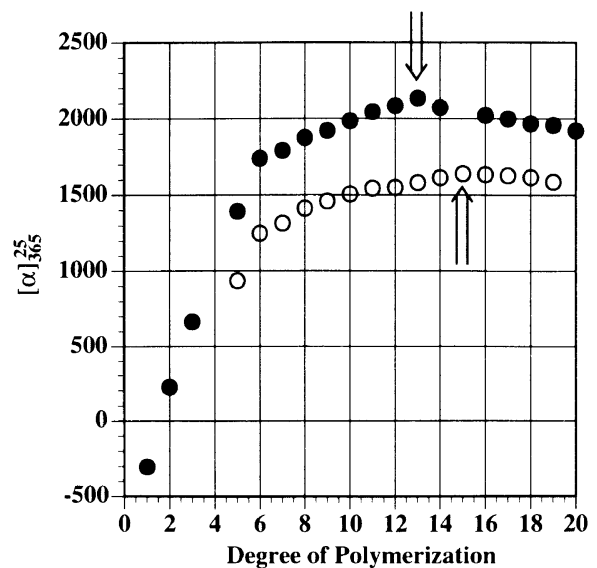

Figure 4. Specific rotation of oligomers of $m \mathrm{MeOPI}()^{-}$and 3,5MePI (O) terminated with $\mathrm{HCl}$-methanol (in THF).

the specific rotation in the higher DP range may be caused by the helix reversal in the main chain. We already reported that in the $m \mathrm{MePI}$ oligomers helix reversal starts from $\mathrm{DP}=13 .{ }^{11}$ From these results, it is clear that 3,5MePI oligomers which have an additional methyl group at the meta position of $m \mathrm{MePI}$ oligomers start to have helix reversals at higher DP than the $m \mathrm{MePI}$ oligomers; in other words, poly(3,5MePI) has a longer average persistence length of a helical structure than poly $(m \mathrm{MePI})$. Both electronic and steric factors of the substituents introduced on the phenyl group may affect the persistence length of the helical structure of these oligomers. In order to examine the electronic effect of the substituent on the phenyl group, we tried to stabilize and isolate the oligomers of $m$ CIPI. The stabilization was performed by introducing an acetyl group at the $\omega$-end.

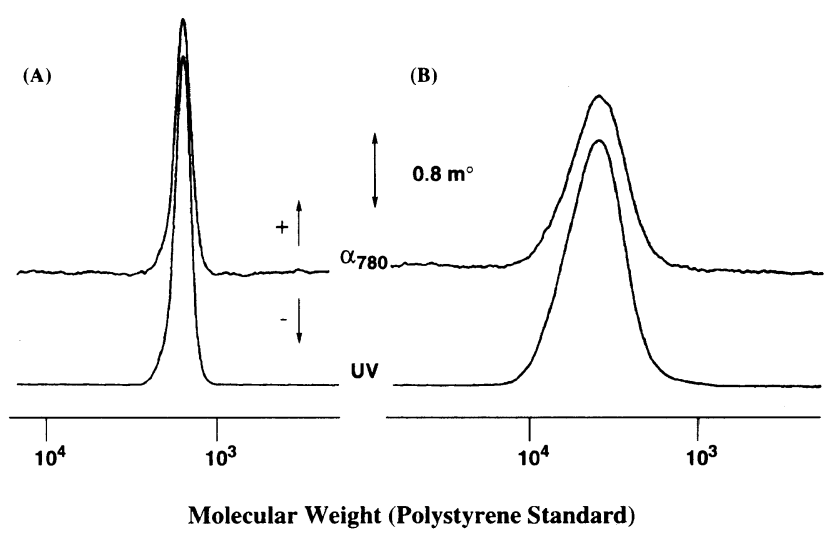

Figure 5. GPC curves of 16-mer (A) and oligomer of $m \mathrm{MeOPI}$ $\left(M_{n}=2.9 \times 10^{3}, M_{w} / M_{n}=1.4,[\alpha]_{365}^{25}+1670^{\circ}\right)(\mathrm{B})$ with polarimeter and UV $(254 \mathrm{~nm})$ detectors.

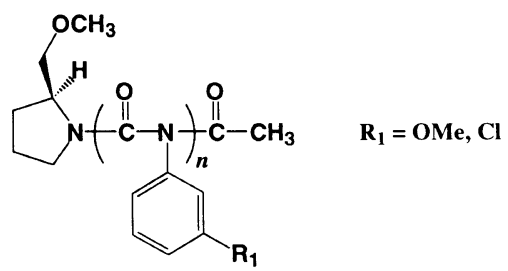

It has already been reported for alkyl isocyanates that acetyl-end-capped oligomers were obtained by the termination with $\mathrm{Ac}_{2} \mathrm{O}$ in the living polymerization system with $\mathrm{TiCl}_{3} \mathrm{OCH}_{2} \mathrm{CF}_{3}$ as initiator and could be separated by SFC. ${ }^{17}$ In order to investigate the effect of the terminal group on the rigidity of the main chain, the oligomerization of $m \mathrm{MeOPI}$ terminated with $\mathrm{Ac}_{2} \mathrm{O}$ was also conducted. The oligomerization reaction of $m \mathrm{ClPI}$ 
and $m \mathrm{MeOPI}$ initiated with $\mathrm{Li}-(-)$-MMP and terminated with $\mathrm{Ac}_{2} \mathrm{O}$ afforded the methanol-insoluble polymers even in the case of $m$ CIPI as shown in Table II. This is in contrast to the results of the oligomerization terminated with $\mathrm{HCl}$-methanol.

In Figure 6, the GPC curves of methanol-soluble part of $m \mathrm{ClPI}$ are shown. The existence of oligomers of higher than $\mathrm{DP}=3$ was observed, indicating that the oligomers of $m$ CIPI were not depolymerized during the oligomerization reaction. The oligo $(m \mathrm{ClPI})$ terminated with $\mathrm{HCl}$-methanol which has an -NH group at the $\omega$-end appears to be easily depolymerized at room temperature. The SFC chromatograms of oligomers of $m \mathrm{ClPI}$ and $m \mathrm{MeOPI}$ terminated with $\mathrm{Ac}_{2} \mathrm{O}$ are shown in Figure 7. In the case of $m \mathrm{MeOPI}$, the $-\mathrm{H}$ end-capped oligomers were removed by depolymerization in DMSO followed by reprecipitation with hexane-ethanol. Therefore, the oligomers of less than DP $=4$ are not observed in Figure 7C. The acetyl-end-capped oligomers of $m \mathrm{ClPI}$ were successfully separated by SFC.

Figure 8 shows the ${ }^{1} \mathrm{H}$ NMR spectrum of 13-mer of $m \mathrm{MeOPI}$ end-capped with acetyl group. The acetyl group introduced at $\omega$-end can be observed at around $2.3 \mathrm{ppm}$. The oligomers were fractionated in terms of DP and the specific rotation of the isolated oligomers was estimated in the same way as described previously.

In Figure 9, the specific rotation of the isolated oligomers of $m \mathrm{ClPI}$ and $m \mathrm{MeOPI}$ end-capped with acetyl

Table II. Oligomerization of meta substituted phenyl isocyanate derivatives with $\mathrm{Li}-(-)-\mathrm{MMP}$ in $\mathrm{THF}$ at $-98^{\circ} \mathrm{C}^{\mathrm{a}}$

\begin{tabular}{cccccc}
\hline & & \multicolumn{4}{c}{ MeOH insoluble part } \\
\cline { 3 - 6 } Run & Monomer & Yield $(\%)^{\mathrm{b}}$ & {$[\alpha]_{365^{25}}{ }^{\mathrm{c}}$} & $M_{n}\left(\times 10^{3}\right)^{\mathrm{d}}$ & $M_{w} / M_{n}^{\mathrm{d}}$ \\
\hline 1 & $m$ ClPI & 3 & $+773^{\circ \mathrm{e}}$ & 3.8 & 1.3 \\
2 & $m$ MeOPI & 34 & $+1860^{\circ} \mathrm{f}$ & 3.3 & 1.4 \\
\hline
\end{tabular}

${ }^{a}$ Conditions: Monomer $0.5 \mathrm{~g}$ (run 1), $1.0 \mathrm{~g}$ (run 2), THF $5 \mathrm{ml}$ (run 1), $10 \mathrm{ml}$ (run 2). $[\mathrm{M}] /[\mathrm{I}]=10$, time $4 \mathrm{~h}$, terminated with acetic anhydride. ${ }^{\mathrm{b}}$ Based on monomer. ${ }^{\mathrm{c}}$ In THF. ${ }^{\mathrm{d}}$ Determined by GPC using polystyrene standard in THF. ${ }^{\mathrm{e}}[\alpha]_{\mathrm{D}}^{25}+189^{\circ} .{ }^{\mathrm{f}}[\alpha]_{\mathrm{D}}^{25}+411^{\circ}$.

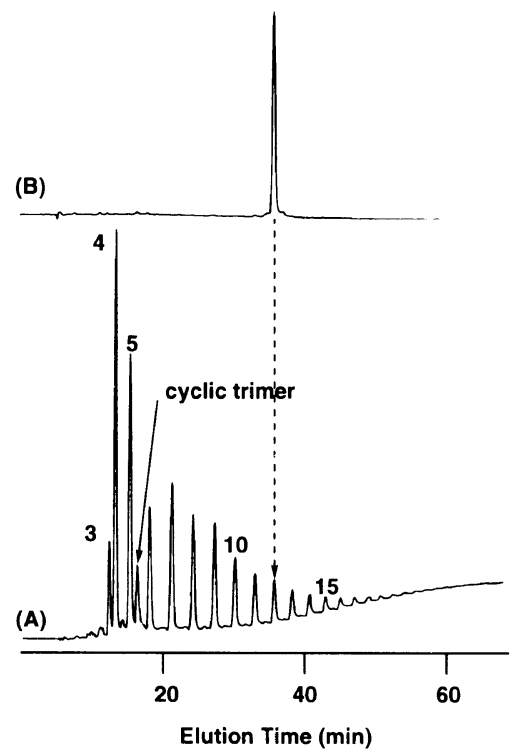

group is plotted against DP. The specific rotation of the acetyl terminated oligomers of $m \mathrm{CIPI}$ and $m \mathrm{MeOPI}$ increased with an increase in DP in the region of DP $\leq$ 10 and $\mathrm{DP} \leq 14$, respectively, and in the higher DP region it gradually decreased with an increase in DP. In the oligomer of $m$ ClPI, helix reversals began to occur at lower DP in comparison with that of $m \mathrm{MeOPI}$ and $m \mathrm{MePI}$, suggesting that the electronic character of the substituent introduced at the meta position affects the persistence length of the helical structure. This may be associated with the fact that the double bond character
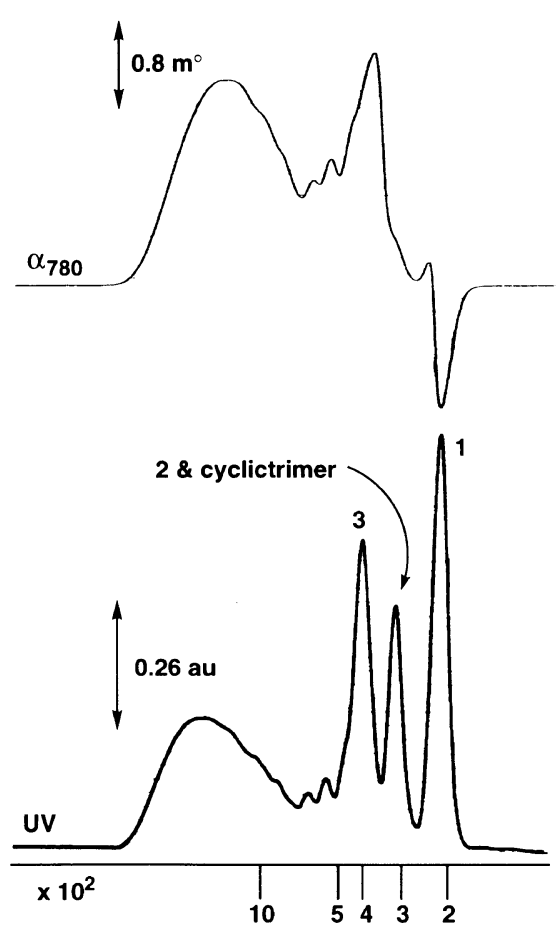

Molecular Weight

(Polystyrene Standard)

Figure 6. GPC curves of oligomer of $m \mathrm{ClPI}$ terminated with acetic anhydride measured with polarimeter (top) and UV (254 $\mathrm{nm})$ (bottom) detectors.

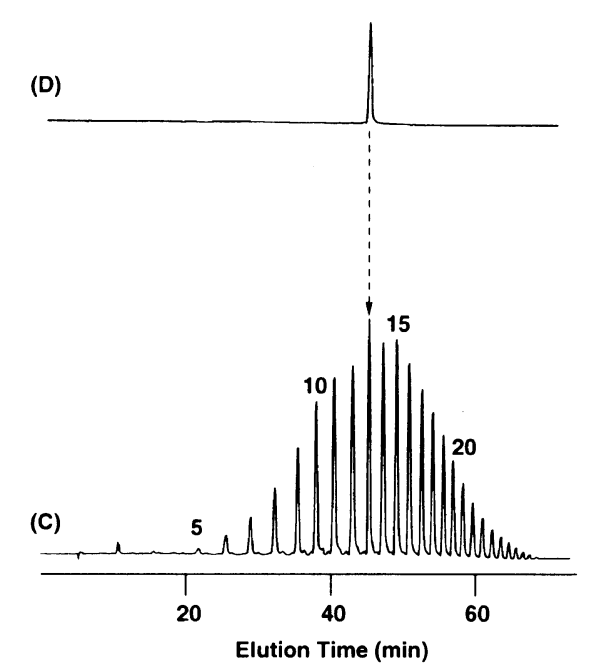

Figure 7. SFC curves of oligo( $m \mathrm{ClPI})(\mathrm{A}), 12$-mer isolated from (A) (B), oligo ( $m \mathrm{MeOPI})(\mathrm{C})$, and 13-mer isolated from (C) (D) (end-capped with acetyl group and measured with UV $(254 \mathrm{~nm})$ detector). 


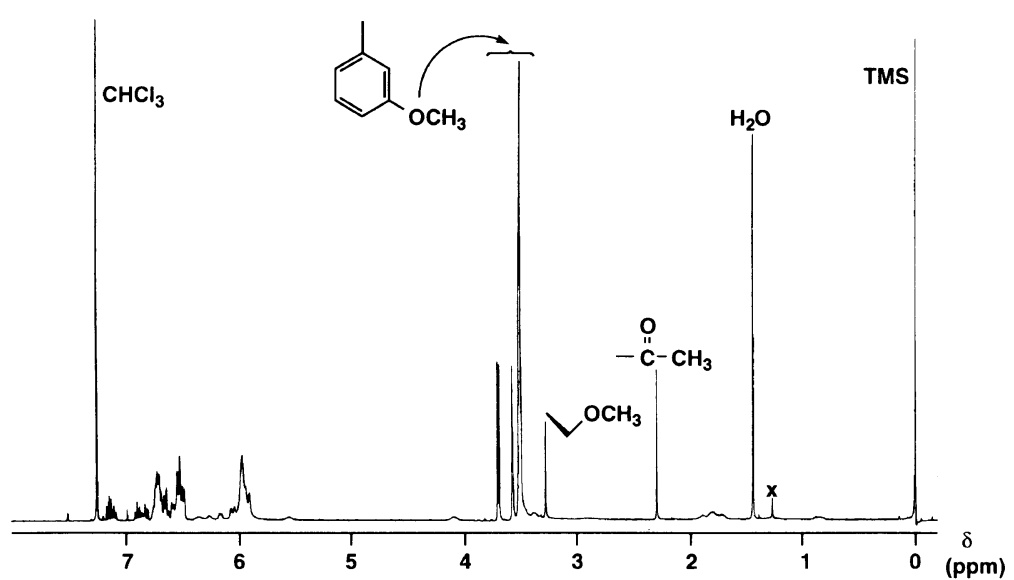

Figure 8. ${ }^{1} \mathrm{H}$ NMR spectrum of 13 -mer of $m \mathrm{MeOPI}$ end-capped with acetyl group $\left(\mathrm{CDCl}_{3}, 60^{\circ} \mathrm{C}, 400 \mathrm{MHz}\right)$.

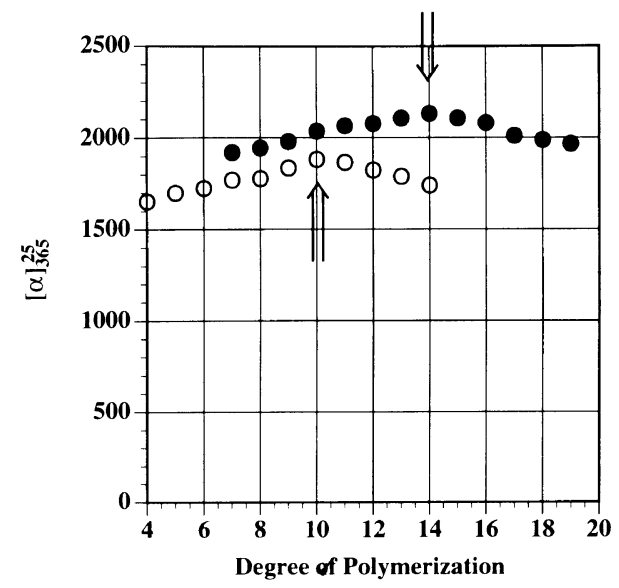

Figure 9. Specific rotation of oligomers of $m \mathrm{MeOPI}(\mathbf{)})$ and $m \mathrm{ClPI}$ (O) end-capped with acetyl group (in THF).

of the main chain of oligo $(m \mathrm{ClPI})$ is reduced by the effect of the electron-withdrawing chloro group. The $-\mathrm{H}$ end-capped oligomers of $m \mathrm{MeOPI}$ starts to have helix reversals at $\mathrm{DP}=13$ (Figure 4) which is slightly lower than that of the acetyl terminated ones. In addition, the acetyl end-capped polymer of $m \mathrm{MeOPI}$ (run 2 in Table II) showed higher specific rotation than the $-\mathrm{H}$ end-capped one (run 1 in Table I) although the acetyl end-capped polymer had a higher-molecular-weight than the $-\mathrm{H}$ end-capped one. These results indicate that the structure of the $\omega$-end of the oligomer also affects the persistence length of the helical structure. In $-\mathrm{H}$ endcapped oligomers, the helix reversal may occur more easily than in acetyl end-capped ones. The less steric hindrance around the $\omega$-end may facilitate the movement of the main chain. The steric effect of acetyl group might be partly canceled by its electron-withdrawing character.

In conclusion, through the chiroptical study on the oligomers of meta substituted phenyl isocyanates having an (S)-2-(methoxymethyl)pyrrolidinyl group at the $\alpha$-end, it becomes clear that poly(aryl isocyanate)s with an electron-withdrawing substituent on the phenyl group show the helix reversal more often than those with an electron-donating substituent and therefore the electronic property of the substituent introduced on the phenyl ring affects the persistence length of the helical structure of the polymer main chain.

Acknowledgment. One of us (K.M.) thanks for the support by a Grant-in-Aid for JSPS Fellows (No. 0574) from the Ministry of Education, Science, Sports, and Culture of Japan. We thank Technical Center of our department for the measurement of FAB mass spectra.

\section{REFERENCES}

1. M. Goodman and S. Chen, Macromolecules, 3, 398 (1970).

2. M. Goodman and S. Chen, Macromolecules, 4, 625 (1971).

3. A. J. Bur and L. J. Fetters, Chem. Rev., 76, 727 (1976).

4. M. M. Green, N. C. Peterson, T. Sato, A. Teramoto, R. Cook, and S. Lifson, Science, 268, 1860(1995).

5. M. M. Green, C. Andreola, B. Muñoz, and M. Reidy, J. Am. Chem. Soc., 110, 4063 (1988).

6. M. M. Green and M. Reidy, J. Am. Chem. Soc., 111, 6452 (1989).

7. Y. Okamoto, M. Matsuda, T. Nakano, and E. Yashima, Polym. J., 25, 391 (1993).

8. M. M. Green, C. Khatri, and N. C. Peterson, J. Am. Chem. Soc., 115, 4941 (1993).

9. C. Khatri, M. M. Vaidya, K. Levon, S. K. Jha, and M. M. Green, Macromolecules, 28, 4719 (1995).

10. Y. Okamoto, M. Matsuda, T. Nakano, and E. Yashima, J. Polym. Sci., A, Polym. Chem., 32, 309(1994).

11. K. Maeda, M. Matsuda, T. Nakano, and Y. Okamoto, Polym. J., 27, 141 (1995).

12. H. Gu, T. Sato, A. Teramoto, L. Varichon, and M. M. Green, Polym. J., 29, 77 (1997).

13. U. Shmueli and W. Traub, J. Polym. Sci., A-2, 7, 515 (1969)

14. K. Ute, Y. Fukunishi, A. Niimi, T. Iwakura, and K. Hatada, Polym. Prepr., Jpn., 45, 3284 (1996).

15. S. Lifson, C. E. Felder, and M. M. Green, Macromolecules, 25, $4142(1992)$

16. A. Teramoto, T. Sato, Y. Nakamura, H. Gu, F. Mukaida, N. Okamoto, M. M. Green, C. Andreola, N. C. Peterson, and S. Lifson, Macromolecules, 29, 2878 (1996).

17. K. Ute, T. Asai, Y. Fukunishi, and K. Hatada, Polym. J., 27, 445 (1995). 